\title{
Radiation and confinement in 0-D fusion systems codes
}

\author{
H. Lux ${ }^{1}$, R. Kemp ${ }^{1}$, E. Fable ${ }^{2}$, R. Wenninger ${ }^{2,3}$ \\ ${ }^{1} \mathrm{CCFE}$, Culham Science Centre, Abingdon, Oxon, OX14 3DB, UK \\ ${ }^{2}$ Max-Plank-Institut für Plasma Physik, D-85748 Garching, Germany \\ ${ }^{3}$ EUROfusion Programme Management Unit, Garching, Germany \\ E-mail: hanni.lux@ukaea.uk
}

\begin{abstract}
In systems modelling for fusion power plants, it is essential to robustly predict the performance of a given machine design (including its respective operating scenario). One measure of machine performance is the energy confinement time $\tau_{E}$ that is typically predicted from experimentally derived confinement scaling laws (e.g. IPB98 $(\mathrm{y}, 2)$ ). However, the conventionally used scaling laws have been derived for ITER which unlike a fusion power plant - will not have significant radiation inside the separatrix.

In absence of a new high core radiation relevant confinement scaling, we propose an ad hoc correction to the loss power $P_{\mathrm{L}}$ used in the ITER confinement scaling and the calculation of the stored energy $W_{\text {th }}$ by

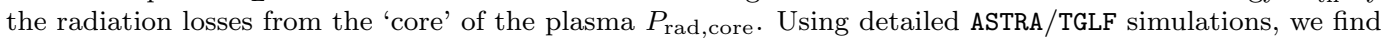
that an appropriate definition of $P_{\text {rad,core }}$ is given by $60 \%$ of all radiative losses inside a normalised minor radius $\rho_{\text {core }}=0.75$. We see is as a zeroth order improvement in current systems modelling and encourage the physics community to work on more accurate predictions.
\end{abstract}

PACS numbers: 52.25.Vy, 52.55.Dy, 52.55.Fa

Keywords: Impurities in plasmas, Magnetic confinement, Tokamak

Submitted to: Plasma Phys. Control. Fusion 


\section{Introduction}

Systems codes are typically used in conceptual design activities for fusion power plants to find optimised design points both consistent with physical laws and technological constraints. For this approach, 0-D models of both the relevant plasma parameters as well as the machine design are employed to quickly navigate through the large parameter space. Within Europe the systems modelling activities are currently mainly focussed on designing a demonstration power plant, DEMO, as specified in the European roadmap to fusion electricity (1).

One central problem of power plant design activities is to find a scenario which has a power balance consistent with the assumed injected heating power and the self-heating power from the fusion reactions. This is normally formulated as

$W_{\text {th }}=P_{\text {heat }} H \tau_{\mathrm{E}, \mathrm{sc}}$

where $W_{\text {th }}$ is the thermal stored energy, $P_{\text {heat }}$ is the net heating power, $H$ is the confinement enhancement $(H)$ factor, and $\tau_{\mathrm{E}, \mathrm{sc}}$ is the predicted energy confinement time obtained from a scaling law. For DEMO studies, the IPB98(y,2)(2) scaling for the confinement time in $\mathrm{s}$ is typically used

$$
\begin{aligned}
\tau_{\text {th }, 98 \mathrm{y} 2}= & 0.0562 I_{\mathrm{p}}^{0.93} B_{\mathrm{t}}^{0.15} n_{1,19}^{0.41} P_{\mathrm{L}}^{-0.69} R_{0}^{1.97} \epsilon^{0.58} \\
& \kappa_{\mathrm{a}}^{0.78} M^{0.19},
\end{aligned}
$$

where $I_{\mathrm{p}}$ is the plasma current in $\mathrm{MA}, B_{\mathrm{t}}$ is the toroidal magnetic field in $T, n_{1,19}$ is the line averaged electron density in $10^{19} \mathrm{~m}^{-3}, P_{\mathrm{L}}$ is the loss power in MW, $R_{0}$ is the machine major radius in $\mathrm{m}, \epsilon=a / R$ is the inverse aspect ratio with the machine minor radius $a, \kappa_{\mathrm{a}}$ is the plasma elongation and $M$ is the ion mass number in atomic mass units.

Unlike current machines a fusion power plant and even ITER will need to reduce the heat load on its divertor plates by a significant amount. The most promising approach to solve this issue is to purposefully seed impurities into the plasma to radiate away the heat and therefore spread it more evenly over the first wall components rather than focus it on a small region in the divertor (e.g. (3)). While ITER is operating already close to its L-H threshold limit and therefore cannot allow any significant radiation from inside the seperatrix, this will be mandatory for DEMO to reduce the load on the divertor to reasonable amounts.

Previous work (4) already raised the issue of how the radiation from the core plasma - that can be seen as a separate loss channel to the conductive/convective losses described in the confinement scaling - should be treated.
Experimentally, Reinke et al. (5) who investigate impurity seeded $\left(\mathrm{N}_{2}, \mathrm{Ne}\right.$ and $\left.\mathrm{Ar}\right)$ enhanced $\mathrm{D}-\alpha$ $\mathrm{H}$-mode experiments on Alcator C-Mod find that energy confinement strongly depends on the difference between the input power and the radiated power inside the last closed flux surface (LCFS). However, the question remains what part of the radiation should be considered as "core radiation" and therefore as instantaneous loss to either/both the heating or loss power in equations 1 and 2 .

Assuming the IPB98 $(y, 2)$ confinement scaling and separating out the dependence on the loss power, a simple parametrisation for the energy confinement can be given as

$$
\begin{aligned}
W_{\mathrm{th}}= & \left(P-\gamma P_{\mathrm{rad}}\right) H\left(P-\chi P_{\mathrm{rad}}\right)^{-0.69} \\
& f_{98}\left(I_{\mathrm{p}}, B_{\mathrm{t}}, n_{\mathrm{l}, 19}, R_{0}, \epsilon, \kappa_{\mathrm{a}}, M\right),
\end{aligned}
$$

where $f_{98}$ summarises all other parameter dependencies of the scaling law. This allows the separation of radiation impact on heating $(\gamma)$ from the effect on confinement $(\chi)$.

A recent international comparison of systems codes (6) showed that as machine parameters generally converge between codes, the calculations of the confinement time and required $H$-factor to achieve a particular performance diverge; a phenomenon which was found to be due to the different treatment of radiation in the different codes. It is clear that there is little agreement on how radiation and confinement should interact in $0-\mathrm{d}$ systems codes. The work in this paper is an attempt to systematically approach the problem.

In Section 2, we describe the method we used to approach this issue using ASTRA/TGLF simulations. We show and discuss our results in Section 3 and conclude in 4 .

\section{Method}

In this work, we assume that $\gamma=\chi$ in equation 3 which seems reasonable if the confinement scaling captures both the energy transport and pedestal characteristics. We furthermore assume that $\gamma$ is dependent on the radiation profile within the plasma - that is, the effect of radiation is dependent on where it is emitted and therefore the calculation of the effective radiation power $\gamma P_{\text {rad }}$ can be reduced to an integral across the profile

$\gamma P_{\text {rad }}=\int f(\rho) P_{\text {rad }}(\rho) d V$

where $\rho$ is the normalised minor radius of the plasma, $f(\rho)$ is a function to be determined which divides the 
radiation at each location into 'core' and 'non-core' radiation and $d V$ is the plasma volume element.

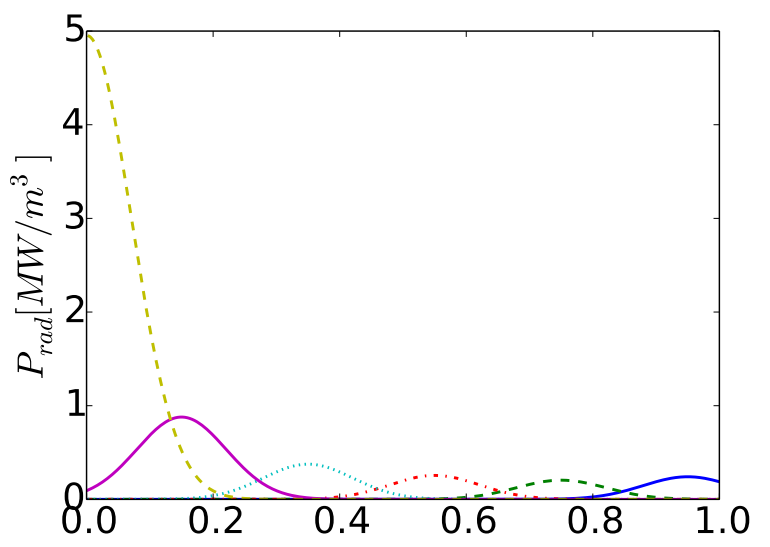

Figure 1. Artificially imposed $\rho$ radiation profiles scanning a range of different peak radiation positions. These profiles have been used as input to our ASTRA/TGLF simulations. The lines shown represent a total radiative power of $P_{\text {rad,tot }} \approx 100 \mathrm{MW}$.

In order to assess the most appropriate form of $f(\rho)$ a series of runs were carried out using the transport code ASTRA $(7 ; 8 ; 9)$, with the core transport coeffcients provided by the predictive model TGLF (10). TGLF has been shown to correctly predict datasets from DIII-D (11) and radiative discharges in ASDEX-Upgrade $(12 ; 13)$. For a typical DEMO reactor configuration, ASTRA/TGLF runs are reasonably consistent with the IPB98 $(\mathrm{y}, 2)$ scaling (see (14) where the full simulations have been described more thoroughly) .

For our analysis, the radiation losses were not chosen to represent any expected/realistic impurity or radiation distributions, but instead were artificially imposed at a range of locations across the plasma. This means also that the radiation behavior is not linked to the impurity content. This decouples the effect on confinement between pure radiation and (eventually) dilution, the latter not studied in this context. As shown in Figure 1 a Gaussian distribution of radiation at 5 different locations with normalised minor radius $\rho=r / a \in[0.1,0.95]$ has been chosen. Additionally, at each location 4 different radiative powers have been assumed $P_{\text {rad,tot }} \in[50-220] \mathrm{MW}$. This allows us to study the effect of both location and radiative power on confinement. All Gaussian distributions have been chosen with the same width and their peak is varied such that the volume integral gives the wanted total radiation.

Please note that these kind of calculations are appropriate in the stiff transport region of the plasma, but that no pedestal height/width scaling has been applied. This kind of consis- tency is beyond the scope of our model and should be kept in mind, when interpreting the results. It is therefore assumed that any systems code separately assures a pedestal consistent with the amount of radiation in the simulation.

\section{Results and Discussion}
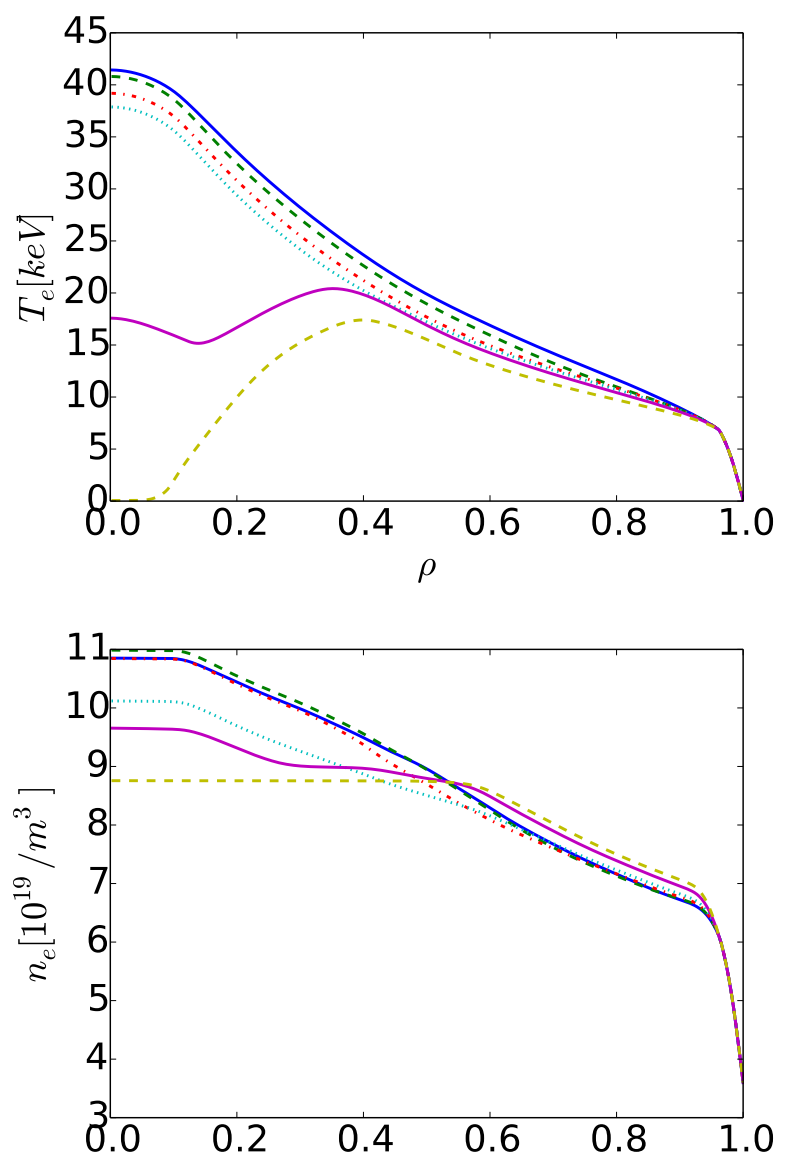

Figure 2. ASTRA/TGLF electron temperature and density profiles resulting from the radiation profiles shown in Figure 1 and $P_{\text {rad,tot }} \approx 100 \mathrm{MW}$. The colour coding and line styles are consistent between all plots. Please note, that the high core radiation cases (dashed, yellow and solid, purple lines) are pathological and beyond the limits of validity of the model. (Note the colour figure are available in the online version only.)

Figure 2 shows the density and temperature profiles resulting from the ASTRA/TGLF runs using the radiation profiles shown in Figure 1. The plots show the case of $P_{\text {rad,tot }} \approx 100 \mathrm{MW}$ and the colours in both figures refer to the same location of the radiation peaks. Please note, that the two cases of the high core radiation push the simulations beyond their limits of validity as significant amounts of power are radiated from the inner regions. This naturally leads to a collapse of the electron temperature at those positions linked to the concurrent reduction in heating from the fusion reaction. 
Assuming that the ITER scaling is correctly capturing the plasma confinement properties with the exception of the instantaneous radiation losses, we can compare it directly to the plasma energy content predicted by the ASTRA/TGLF runs to derive an appropriate correction to the loss power as suggested in equation 4. In lack of a good intuition for what the form of $f(\rho)$ should be, we test 4 potential models covering a range of different behaviours. Our first model assumes a linear function

$f_{1}(\rho)=C_{1}(1-\rho)$,

where $C_{1}$ is a free fitting parameter. As an alternative, one could imagine a square relation with the normalised minor radius $\rho$ as given by

$f_{2}(\rho)=C_{2}\left(1-\rho^{2}\right)^{\alpha}$,

where $\alpha$ is a peaking parameter and $C_{2}$ again a free scaling parameter. As a third, model we suggest a simple step function, that defines a core radius $\rho_{\text {core }}$ within which all radiation is considered as instantaneous losses that should be subtracted from the loss power. This suggest $f(\rho)$ has the following form

$f_{3}(\rho)=C_{3} \begin{cases}1 & \text { if } \rho \leq \rho_{\text {core }} \\ 0 & \text { if } \rho>\rho_{\text {core }}\end{cases}$

This model is relatively easy to interpret despite being rather crude in assuming that an abrupt cut off radius is physical meaningful. As a last, model we use a square root scaling

$f_{4}(\rho)=C_{4} \sqrt{1-\rho}$

where $C_{4}$ is a again a free fitting parameter.

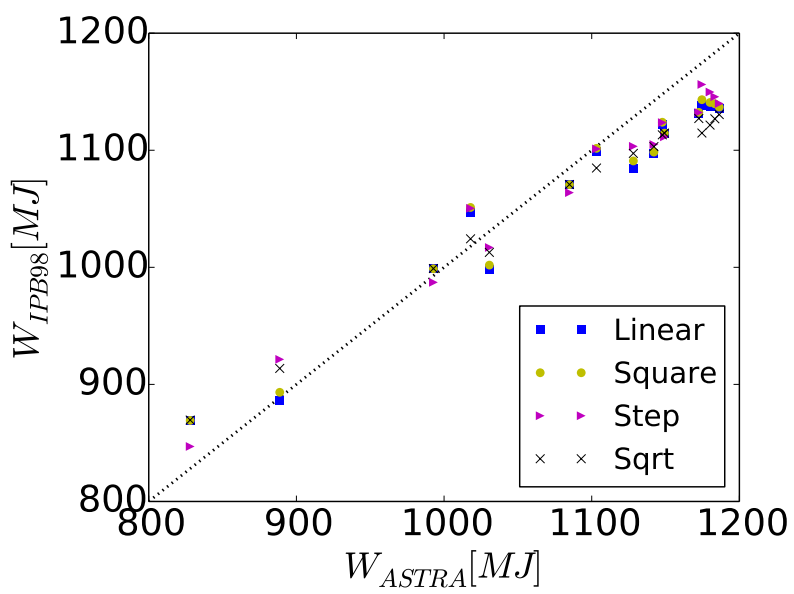

Figure 3. Plot of calculated stored energy from the IPB98 $(y, 2)$ scaling versus the stored energy calculated from ASTRA/TGLF for different choices of $f(\rho)$ excluding pathological cases as e.g. the dashed, yellow and solid, purple lines shown in Figure 2. These are labelled as: eq. (5) Linear; (6) Square; (7) Step; (8) Sqrt.
In Figure 3, we compare the plasma energy content $W_{\text {ASTRA }}$ in the ASTRA/TGLF runs with the predictions from the IPB98(y,2) scaling law $W_{\text {IPB98 }}$ assuming corrections to the loss power as given by our four suggested models for $f(\rho)$. The symbols indicate the data points from the respective best fits for each model and the straight line shows a one-to-one correlation. Table 1 summarises the best fit parameters and the corresponding coefficient of determination $R^{2}$ of our least squares analysis. Even though the absolute values of $R^{2}$ seem to indicate that the 'step' function gives the best fit in comparison to the other models, all four models fit the data reasonably well and can be equivalently used in correcting the IPB98(y,2) scaling law for high core radiation scenarios.

\begin{tabular}{|c|c|c|c|}
\hline Fit & Eq. & Parameters & $R^{2}$ \\
\hline Linear & $(5)$ & $C_{1}=1.2$ & 0.89 \\
Square & $(6)$ & $C_{2}=0.9, \alpha=1.4$ & 0.90 \\
Step & $(7)$ & $C_{3}=0.6, \rho_{\text {core }}=0.75$ & 0.92 \\
Sqrt & $(8)$ & $C_{4}=0.8$ & 0.87 \\
\hline
\end{tabular}

Table 1. Best fit parameters for the different models.

However, we have noted before that the ASTRA results are not really valid for the high radiation peaks in the core region nor any radiation peaks in the pedestal region. Therefore, it makes sense to opt for a model for $f(\rho)$ that does not rely on robust data in either of these regions. Based on this argument and the intuitive interpretation of the step model, we therefore recommend correcting the loss power in the IPB98 scaling law (eq. 2) as well as in the calculation of the stored energy (eq. 1) by the power radiated within $\rho_{\text {core }}=0.75$ with a normalisation factor of $C_{3}=0.6$.

Figure 4 shows the detailed least squares analysis for the step function. It illustrates the degeneracy of the results. Suggesting that other combinations of values for $\rho_{\text {core }}=0.75 \pm 0.05$ and $C_{3}=0.6 \pm 0.1$ are also reasonably valid. Please note that in a preliminary analysis referenced in (15) we did not exclude the pathological cases. This lead to a result closer to $\rho_{\text {core }}=0.6$ and $C_{3}=1$. The cyan squares in Figure 4 indicate the $R^{2}$ values of this solution and show that they still fit the data reasonably well, even though they are not nominally the best fit anymore.

\section{Conclusions}

Within systems modelling, confinement scaling laws are an essential tool to predict the performance of (demonstration) fusion power plants like the European DEMO. However, the typically used ITER confinement scaling law IPB98 $(\mathrm{y}, 2)$ for ELMy $\mathrm{H}$-modes has been derived from an experimental database that unlike 


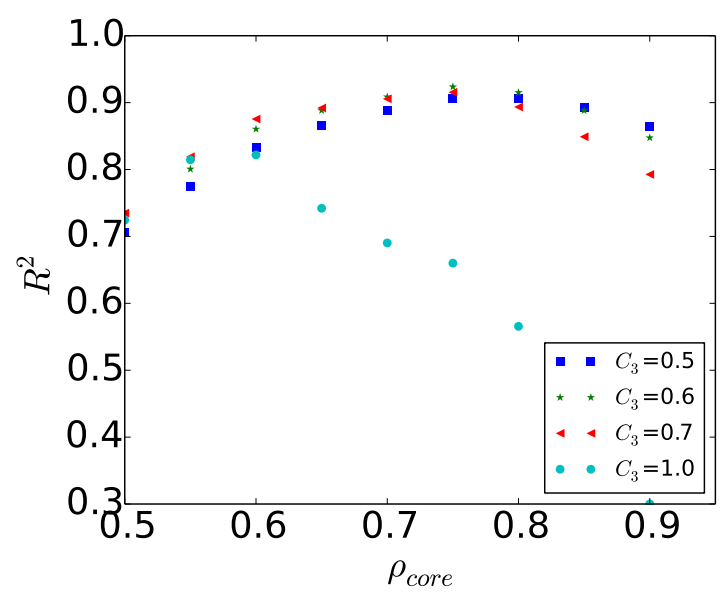

Figure 4. Plot of the coefficient of determination $R^{2}$ of our least squares analysis for the 'step' fitting function 7 vs. the two fit parameters $C_{3}$ and $\rho_{\text {core }}$ illustrating the robustness of the best fit parameters given in table 1 .

fusion power plants has low total and especially low core radiation. Assuming that the scaling law can still be used to extrapolate to DEMO like machines, if the loss power is corrected by instantaneous losses, we tests several models for location dependent radiation corrections. In comparing the actual stored energies to predicted stored energies from the different corrections of the IPB98 $(\mathrm{y}, 2)$ scaling in ASTRA/TGLF runs with different radiation distributions and powers, we find that the most appropriate model is subtracting $60 \%$ of all radiative losses inside a normalised minor radius $\rho_{\text {core }}=0.75$ from the heating power. This leads to

$P_{L} \equiv P_{\text {heat }}=P-0.6 \int_{\rho=0}^{0.75} P_{\mathrm{rad}}(\rho) d V$

in equations 1 and 2 , where $P$ represents the total heating power given by both the $\alpha$ and any auxiliary heating. The result from a preliminary analysis that suggested subtracting all radiative power within $\rho_{\text {core }}=0.6$ is still yields acceptable results given the other uncertainties in the model.

\section{Acknowledgements}

It is a pleasure to thank David Ward and Martin O'Brien for encouragement and stimulating discussions. This work has been (part-)funded by the RCUK Energy Programme [grant number EP/I501045]. To obtain further information on the data and models underlying this paper please contact PublicationsManager@ccfe.ac.uk. Furthermore, this work has been carried out within the framework of the EUROfusion Consortium and has received funding from the Euratom research and training programme 2014-2018 under grant agreement No 633053. The views and opinions expressed herein do not necessarily reflect those of the European Commission.

\section{References}

[1] Romanelli F 2012 Fusion electricity - a roadmap to the realisation of fusion energy EFDA

[2] on Confinement I P E G, Transport, on Confinement Modelling I P E G, Database and Editors I P B 1999 Nuclear Fusion 392175 URL http://stacks.iop.org/0029-5515/39/i=12/a=302

[3] Wischmeier M 2014 Journal of $\mathrm{Nu}$ clear Materials - ISSN 0022-3115 URL http://www.sciencedirect.com/science/article/pii/S00223115

[4] Ward D J 2010 Plasma Physics and Controlled Fusion $\mathbf{5 2} 124033 \quad$ URL http://stacks.iop.org/0741-3335/52/i=12/a=124033

[5] Reinke M, Hughes J, Loarte A, Brunner D, Hutchinson I and et al 2011 Journal of Nuclear Materials 415 S340 - S344 ISSN 0022-3115 URL http://www.sciencedirect.com/science/article/pii/S00223115

[6] Ward D J, Chan V, Kemp R, Kessel C, Nakamura M, Srinivasan R, Reux C, Vinay M and Morris J 2013 International systems code benchmark for demo IAEA DEMO Programme Workshop, Vienna, Session 1-5

[7] Fable E, Angioni C, Casson F J, Told D, Ivanov A A, Jenko F, McDermott R M, Medvedev S Y, Pereverzev G V, Ryter F, Treutterer W, Viezzer E and the ASDEX Upgrade Team 2013 Plasma Physics and Controlled Fusion 55124028 URL http://stacks. iop.org/0741-3335/55/i=12/a=124028

[8] Fable E, Angioni C, Ivanov A, Lackner K, Maj O, Yu S, Medvedev, Pautasso G and Pereverzev G 2013 Nuclear Fusion $\mathbf{5 3} 033002$ URL http://stacks. iop.org/0029-5515/53/i=3/a=033002

[9] Pereverzev G and Yshmanov Y 1991 Tech. Rep. 5/42 IPP aSTRA documentation

[10] Staebler G M, Kinsey J E and Waltz R E 2007 Physics of Plasmas (1994-present) $\mathbf{1 4} 055909$ URL http://scitation.aip.org/content/aip/journal/pop/14/5/10.1

[11] Kinsey J E, Staebler G M and Waltz R E 2008 Physics of Plasmas 15055908 URL http://scitation.aip.org/content/aip/journal/pop/15/5/10.1

[12] Sommer F, Stober J, Angioni C, Fable E, Orte L B, Bernert M, Bobkov $\mathrm{V}$ and et al 2012 H-mode characterisation for dominant ecrh and comparison to dominant nbi heating at asdex upgrade 39th EPS Conference 8 16th Int. Congress on Plasma Physics 
vol Vol. 36F (European Physical Society) URL

ocs.ciemat.es/epsicpp2012pap/pdf/P5.085.pdf

[13] Sommer F, Stober J, Angioni C, Fable E, Bernert M, Burckhart A, Bobkov V, Fischer R, Fuchs C, McDermott R, Suttrop $\mathrm{W}$, Viezzer $\mathrm{E}$ and the ASDEX Upgrade Team 2015 Nuclear Fusion 55033006 URL http://stacks. iop.org/0029-5515/55/i=3/a=033006

[14] Fable E, Wenninger R and Kemp R 2016 submitted to Nuclear Fusion

[15] Lux H, Kemp R, Ward D and Sertoli M 2015 Fusion Engineering and De$\begin{array}{llllllll}\text { sign } & 101 & 42 & - & 51 & \text { ISSN } & 0920-3796 & \text { URL }\end{array}$ http://www.sciencedirect.com/science/article/pii/S0920379615302891 